\title{
Dynamics of Equilibrium Points in a Uniformly Rotating Second-Order and Degree Gravitational Field
}

\author{
Jinglang Feng ${ }^{1,2}$ and Xiyun Hou ${ }^{1,2}$ \\ ${ }^{1}$ School of Astronomy and Space Science, Nanjing University, 210093, China; jinglang@ nju.edu.cn \\ ${ }^{2}$ Institute of Astrodynamics and Space Environment, Nanjing University, 210093, China; silence@nju.edu.cn \\ Received 2017 January 25; revised 2017 April 9; accepted 2017 May 18; published 2017 June 23
}

\begin{abstract}
Using tools such as periodic orbits and invariant manifolds, the global dynamics around equilibrium points (EPs) in a rotating second-order and degree gravitational field are studied. For EPs on the long axis, planar and vertical periodic families are computed, and their stability properties are investigated. Invariant manifolds are also computed, and their relation to the first-order resonances is briefly discussed. For EPs on the short axis, planar and vertical periodic families are studied, with special emphasis on the genealogy of the planar periodic families. Our studies show that the global dynamics around EPs are highly similar to those around libration points in the circular restricted three-body problem, such as spatial halo orbits, invariant manifolds, and the genealogy of planar periodic families.
\end{abstract}

Key words: instabilities - methods: numerical - minor planets, asteroids: general

\section{Introduction}

In recent years, there has been a growing interest in space missions targeting small bodies in the solar system, as these bodies not only provide us with clues on the formation and evolution of our solar system but are also of potential economic value. For example, the Hayabusa spacecraft touched down on the asteroid Itokawa and, for the first time, returned samples of regolith to Earth (Fujiwara et al. 2006). After visiting Vesta, the Dawn spacecraft is now orbiting Ceres, exploring its surface geology and interior structure (Russell et al. 2012). Recently, the Rosetta spacecraft ended its mission with an impact on the surface of its target body: the comet 67P, from which a huge amount of scientific information about comets was uncovered (Sierks et al. 2015). For missions to these small bodies, one challenge is their weak and irregular gravitational fields due to their small sizes and irregular shapes. For an asteroid with a relatively large size and an orbit close to its surface (within several radii of the asteroid), the asteroid's gravity usually dominates. For an asteroid of small size or orbital motions far away from it, the solar radiation pressure may be stronger (Scheeres 2012; Broschart et al. 2014).

To model the irregularity of the gravitational field, several ideas have been proposed, such as the mascon model (Geissler et al. 1996) and the polyhedron model (Werner \& Scheeres 1997). But even if we can accurately compute the gravitational field with these models, along with the precise orbits around them (Yu \& Baoyin 2012), an orbit found in the vicinity of one asteroid generally cannot be directly applied to another one due to the specific shape of each individual asteroid. To gain some general insights on the dynamics around an irregular body, a simplified shape model is usually applied to represent the gravitational field, also with less computation cost, such as the gravitational field of a bar (Elipe \& Lara 2004), contact binaries (Feng et al. 2015), and several spheres or ellipsoids with different configurations (Zeng et al. 2015). Among these simplified shapes, a uniform triaxial ellipsoid is applied in many studies due to its simplicity and closed form (Chauvineau et al. 1993). With this model, Scheeres (1994) investigated the orbital dynamics, with special emphasis on the equilibrium points (EPs) in the frame rotating with the ellipsoid. Assuming a uniform rotation for the ellipsoid, there are four EPs, two of which lie on the long axis (LEPs) and two of which lie on the short axis (SEPs). The LEPs are always unstable, while the stability of the SEPs depends on the ellipsoid's shape and rotation speed.

Another traditional approach to representing nonspherical gravitational fields is the spherical harmonics expansion. It has been widely applied to studying the orbital dynamics around asteroids (Scheeres et al. 2000; Hu \& Scheeres 2002; Tricarico \& Sykes 2010; Delsate 2011; Ceccaroni \& Biggs 2013; Feng et al. 2017) due to its simplicity and the fact that it can be explicitly associated with changes of orbital elements analytically (Kaula 1966). In this work, we use the gravitational field truncated at the second order and second degree (2OD), which captures the dominant part of the nonspherical terms and is capable of studying the qualitative behaviors of orbits (Scheeres 1999; Scheeres \& Hu 2001; Hu \& Scheeres 2004). However, for some odd-shaped asteroids or orbits very close to the asteroids' surface (Magri et al. 2007; Wang et al. 2014), high-order terms may need to be considered. For asteroids of small sizes, solar radiation pressure is also important and should be taken into consideration (Xin et al. 2016); this is outside the scope of this paper.

This work is devoted to the dynamics around EPs in a rotating 2OD gravitational field, with asteroids as the research targets. Compared with previous studies (Scheeres 1994; Vasilkova 2005; Jiang et al. 2014), which mainly focus on the linear stability of the EPs and the motions in their vicinity, the global dynamics around the EPs are studied in this work by computing periodic orbits (POs) and the invariant manifolds associated with them, with special focus on the genealogy and stability of the periodic families. Since the orbits around the EPs are also resonance orbits, which are in 1:1 resonance with the asteroid's rotation in the inertial frame rather than the EPs themselves, this work can be treated as a study of the 1:1 resonance orbits but carried out in a rotating frame with the tools of dynamical systems.

The contents of this work are as follows. Section 2 lays the foundations for the current study, including equations of motion (EOMs) in the 2OD gravity, the Jacobi constant, EPs, and the methodology for computing periodic families. 
In Section 3, the dynamics around the LEPs are investigated. The following results are found. (1) For slowly rotating asteroids, stable planar Lyapunov orbits that do not collide with the asteroid's surface exist. They are generally of large amplitudes and are actually highly eccentric 1:1 resonance orbits in the asteroid's equatorial plane, with the periapse and apoapse aligning along the asteroid's long axis. (2) For highly irregular asteroids, spatial POs generated from the planar Lyapunov family around the LEP also exist, similar to the circular restricted three-body problem (CRTBP), where halo orbits are generated from the planar Lyapunov family around the collinear libration points. (3) The reachable region of the invariant manifolds associated with the LEP is closely connected with the inner and outer resonances, a phenomenon also similar to the CRTBP. (4) Members of the vertical Lyapunov family are generally unstable, which means that inclined 1:1 resonance orbits with the ascending node along the long axis are unstable.

In Section 4, the dynamics around the SEPs are investigated. Studies show several points. (1) The planar long-period family terminates onto a planar short-period orbit traveling $N$ times, where the value of $N$ depends on the two basic frequencies in the linearized model of the planar motions around the SEPs. From this same $N$-bifurcation, a short-period orbit generates another periodic family, connecting it with an $(N+1)$-bifurcation short-period orbit. From this $(N+1)$-bifurcation short-period orbit, yet another periodic family bifurcates, connecting it with an $(N+2)$-bifurcation short-period orbit. This genealogy is exactly the same as that of the planar long- and short-period families around the triangular libration points in the CRTBP (Hou \& Liu 2009). (2) With the rotation speed increasing, the stability of the SEPs changes from "stable" to "unstable." Meanwhile, the planar long- and shortperiod families merge to form a single family. In this case, even though the SEPs are unstable, stable POs of finite sizes still exist around them. This is also similar to the case of the triangular libration points of the CRTBP (Hou \& Liu 2009). (3) The stability of the vertical Lyapunov family depends on the specific values of $C_{20}$ and $C_{22}$ in the 2OD gravitational field.

Finally, two remarks are made. (1) Hovering above an asteroid is an important approach to exploring it, which is different from the approach of cycling it. The EPs are ideal places for probes to hover above asteroids, so it is useful to carry out studies on the global dynamics around the EPs. (2) To gain some general insights and conclusions on the global dynamics around the EPs of uniformly rotating asteroids, we carry out the work in the 2OD gravitational field and neglect higher-order nonspherical terms for specific asteroids. Nevertheless, it would be interesting to carry out similar work for specific asteroids and compare the results with the general conclusions in this work. The comparison may show some differences for orbital motions close to the asteroid's surface. The results in the current work can be used as good initial guesses about POs in the true gravitational fields of specific asteroids.

\section{Dynamical Modeling}

\subsection{Equations of Motion}

In the body-fixed frame of the asteroid, the EOMs of a massless body are

$$
\ddot{\boldsymbol{r}}+2 \boldsymbol{\omega}_{a} \times \dot{\boldsymbol{r}}+\boldsymbol{\omega}_{a} \times\left(\boldsymbol{\omega}_{a} \times \boldsymbol{r}\right)=\frac{\partial V}{\partial \boldsymbol{r}}
$$

where $\omega_{a}$ is the asteroid's rotation rate and $V$ is minus the gravity potential. As mentioned in Section 1, we take the approach of spherical harmonics to express the asteroid's gravitational potential. It takes the following form (Kaula 1966):

$$
\begin{aligned}
& V=\frac{G M}{r} \\
& \left\{1+\sum_{n \geqslant 1}^{\infty} \sum_{m=0}^{n}\left(\frac{R_{e}}{r}\right)^{n} P_{n m}(\sin \theta)\left[C_{n m} \cos m \lambda+S_{n m} \sin m \lambda\right]\right\},
\end{aligned}
$$

in which $G M$ is the gravitational constant of the asteroid, $(\lambda, \theta)$ are the longitude and latitude, $C_{n m}$ and $S_{n m}$ are the Stokes coefficients, $P_{n m}$ is the associated Legendre function, and $R_{e}$ is the reference radius of the asteroid. In our work, we choose

$$
R_{e}=r_{\mathrm{syn}}=\left(G M / \omega_{a}^{2}\right)^{1 / 3}
$$

which is the radius of the synchronous orbit by taking the asteroid as a particle. The mass and time units are chosen as the mass of the asteroid and $1 / \omega_{a}$, respectively. After normalization of units, Equation (1) becomes

$$
\ddot{x}-2 \dot{y}-x=\frac{\partial V}{\partial x}, \ddot{y}+2 \dot{x}-y=\frac{\partial V}{\partial y}, \ddot{z}=\frac{\partial V}{\partial z},
$$

and Equation (2) becomes

$$
\begin{aligned}
& V=\frac{1}{r} \\
& \left\{1+\sum_{n \geqslant 1}^{\infty} \sum_{m=0}^{n}\left(\frac{1}{r}\right)^{n} P_{n m}(\sin \theta)\left[C_{n m} \cos m \lambda+S_{n m} \sin m \lambda\right]\right\} .
\end{aligned}
$$

One remark is made here. Usually, the reference radius $R_{e}$ is fixed for an asteroid and is chosen as a value that is associated with the asteroid's size, such as its equatorial axis. The associated Stokes coefficients are constants independent of the asteroid's rotation. In this sense, our choice of $R_{e}$ by Equation (3) is extraordinary, and the associated Stokes coefficients vary with the asteroid's rotation period. The advantages are twofold: (1) we have a minimum set of parameters, $C_{20}$ and $C_{22}$, thus excluding other parameters such as size, mass, and rotation period of the asteroid; and (2) the same set of values of $C_{20}$ and $C_{22}$ corresponds to different physical systems (see Equation (9)). Thus, changes in the values of $C_{20}$ and $C_{22}$ can be interpreted as changes in either the asteroid's shape and size or its rotation period. This makes the current study with a fixed set of values of $C_{20}$ and $C_{22}$ applicable to a group of asteroids instead of just one. The disadvantage is that it is difficult to identify whether or not the orbit collides with the asteroid based only on the $C_{20}$ and $C_{22}$ values, due to the exclusion of the asteroid's physical size. As a result, in the following, if we have to consider the physical space (i.e., whether or not the orbit collides with the asteroid), we have to attribute another two parameters: the asteroid's rotation period and physical size. 

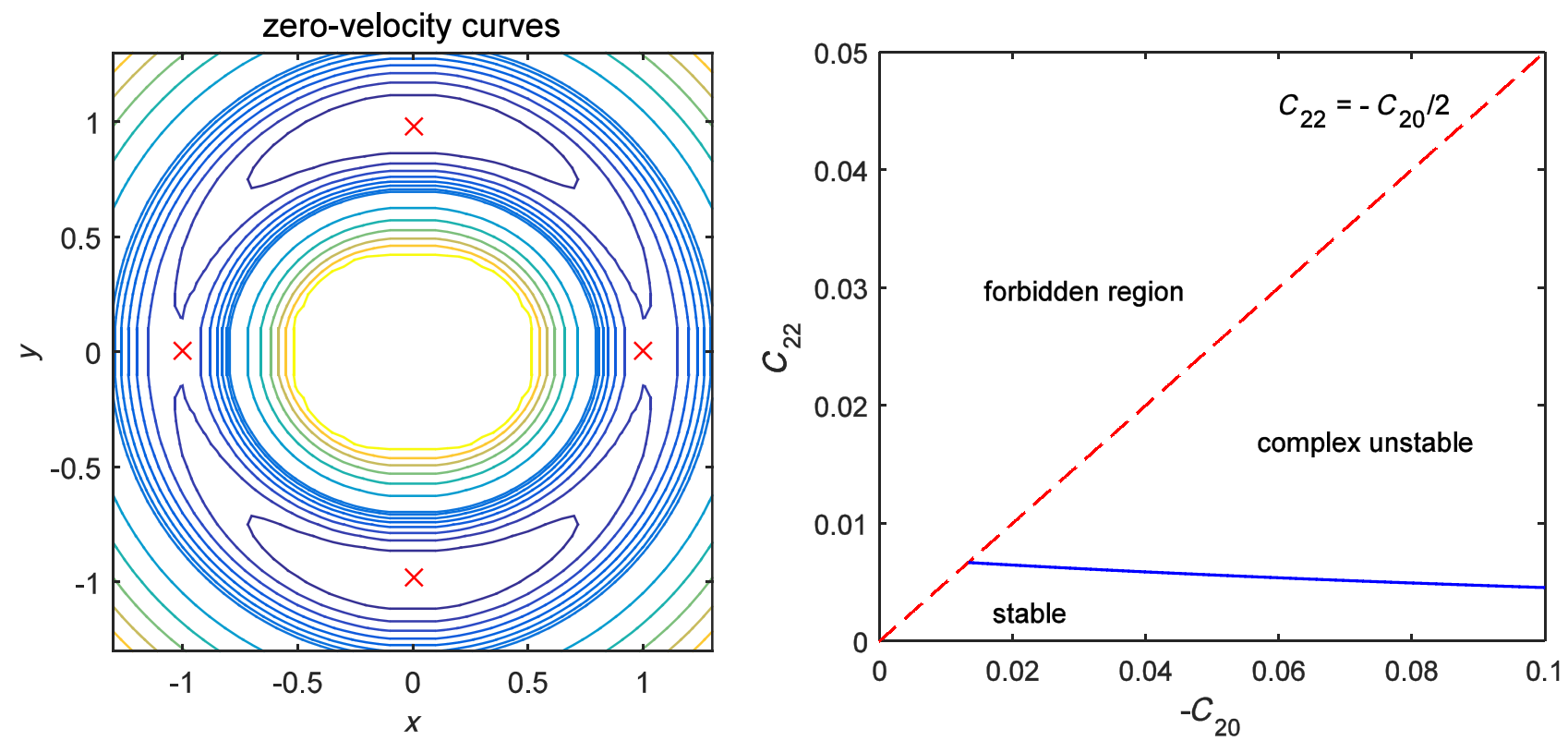

Figure 1. Left: ZVCs and positions of four EPs (red crosses) of a 2OD gravitational field in the $x-y$ plane. Right: stability region of a SEP in the $C_{20}-C_{22}$ plane.

Equation (4) admits an integral in the form of

$$
C=T_{\text {kinetic }}-V^{\prime}=\frac{\left(\dot{x}^{2}+\dot{y}^{2}+\dot{z}^{2}\right)}{2}-\left(V+\frac{x^{2}+y^{2}}{2}\right) \text {, }
$$

which we also call the Jacobi integral because it has the same form as that of the CRTBP. Here $C$ is the Jacobi constant and $V^{\prime}$ is the effective potential in the rotating frame. Truncated at the second order, we have (we can always choose the coordinates of the body-fixed frame to make $S_{22}=0$ )

$$
\begin{aligned}
V & =\frac{1}{r}\left\{1+\left(\frac{1}{r}\right)^{2} C_{20} P_{2}(\sin \theta)+\left(\frac{1}{r}\right)^{2} P_{22}(\sin \theta) C_{22} \cos 2 \lambda\right\} \\
& =\frac{1}{r}+\frac{C_{20}}{r^{3}}\left(\frac{3}{2} \frac{z^{2}}{r^{2}}-\frac{1}{2}\right)+\frac{3 C_{22}}{r^{5}}\left(x^{2}-y^{2}\right) .
\end{aligned}
$$

To attribute physical meaning to the values of $C_{20}$ and $C_{22}$, we assume a triaxial model for the asteroid with three semiaxes as $a \geqslant b \geqslant c$ and a constant density of $2.5 \mathrm{~g} \mathrm{~cm}^{-3}$. We have (Balmino 1994)

$$
C_{20}=\frac{1}{5 R_{e}^{2}}\left(c^{2}-\frac{a^{2}+b^{2}}{2}\right), \quad C_{22}=\frac{1}{20 R_{e}^{2}}\left(a^{2}-b^{2}\right) .
$$

Denoting $\alpha=b: a, \beta=c: a$ and substituting Equation (3) into Equation (8), we have

$$
\begin{aligned}
& C_{20}=\frac{1}{10}\left(\frac{\omega_{a}^{2}}{G \rho}\right)^{2 / 3} \frac{\left(2 \beta^{2}-\alpha^{2}-1\right)}{(\alpha \beta)^{2 / 3}}, \\
& C_{22}=\frac{1}{20}\left(\frac{\omega_{a}^{2}}{G \rho}\right)^{2 / 3} \frac{\left(1-\alpha^{2}\right)}{(\alpha \beta)^{2 / 3}},
\end{aligned}
$$

where $\rho$ is the density. From Equation (9), we know that $C_{20}$ and $C_{22}$ are up to the asteroid's rotation speed (and density) and shape. Ignoring the density, there are two ways to change the values of
$C_{20}$ and $C_{22}$. One is to change the asteroid's rotation speed, and the other is to change its shape. The two ways are equivalent to each other, as long as they have the same values of $C_{20}$ and $C_{22}$. One special remark is made. Although we use the triaxial ellipsoid shape model for the asteroid, we only consider the 2OD gravitational potential of the ellipsoid, not the full gravitational potential. The aim of the current study is to get some general insights on the orbital motion in the 2OD gravitational potential. The only purpose of employing the ellipsoid shape model here is to help us easily calculate the values of $C_{20}$ and $C_{22}$ and visualize the relative size of the orbits with respect to the asteroid (i.e., whether or not they collide with it). As mentioned at the end of Section 1, further studies are necessary if we want to deal with true orbital motions around specific asteroids, even if they are really in the shapes of ellipsoids. In this case, results in the 2OD gravitational potential can serve as good initial guesses as to the orbital motions in the asteroid's real gravitational field.

Similar to the CRTBP, $C=V^{\prime}$ also defines a zero-velocity surface that divides the phase space into accessible regions $\left(T_{\text {kinetic }} \geqslant 0\right)$ and forbidden regions $\left(T_{\text {kinetic }}<0\right)$. When the motion is restricted in the $x-y$ plane (i.e., $z \equiv 0$ ), we have zerovelocity curves (ZVCs). Taking the asteroid with $a=1 \mathrm{~km}$, $b=0.6 \mathrm{~km}, c=0.4 \mathrm{~km}$, and a rotation period of $12 \mathrm{hr}$ as an example, Figure 1 shows the ZVCs at different Jacobi constants.

\subsection{Equilibrium Points}

EPs appear as fixed points in the asteroid's body-fixed frame. They can be interpreted as exact 1:1 resonance orbits around the asteroid in the inertial frame. Truncated at the 2OD gravitational potential, there are four EPs outside the asteroid. Two of them lie on the $x$-axis (LEPs), and the other two lie on the $y$-axis (SEPs). Their positions can be obtained by setting $\dot{x}=\ddot{x}=\dot{y}=\ddot{y}=\dot{z}=\ddot{z}=0$ in Equation (4). Denoting the 


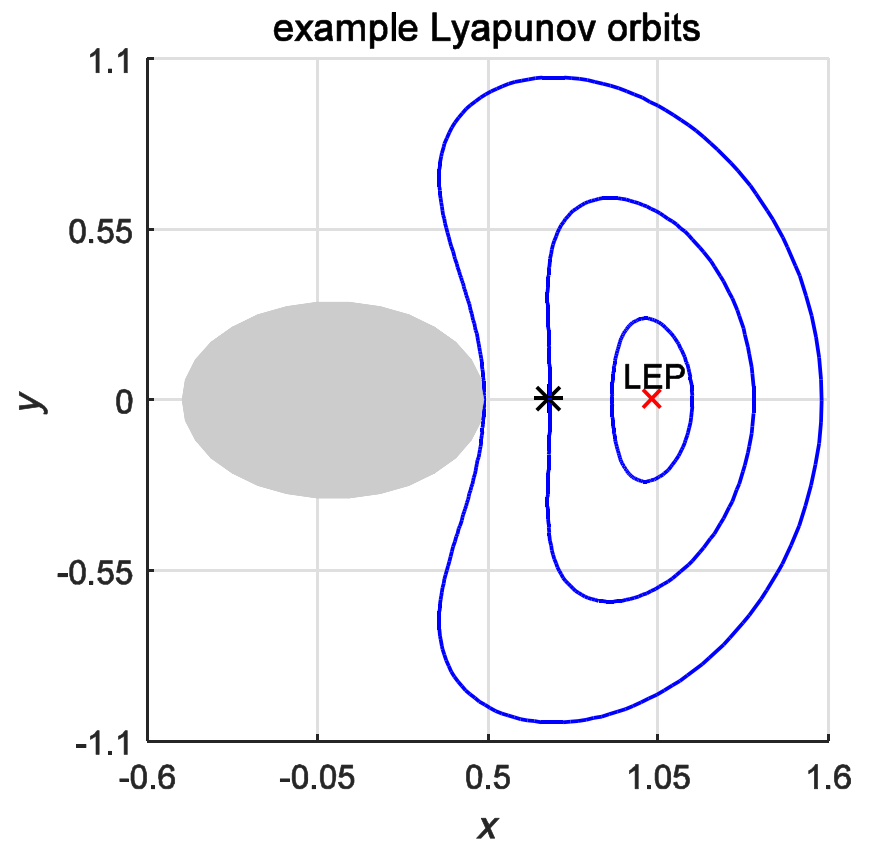

Figure 2. Example orbits of the planar Lyapunov family.

distance of the EPs from the origin as $r_{0}$, we have

$$
\begin{cases}1-\frac{1}{r_{0}^{3}}+\frac{3 C_{20}}{2 r_{0}^{5}}-\frac{9 C_{22}}{r_{0}^{5}}=0, & \text { for LEP } \\ 1-\frac{1}{r_{0}^{3}}+\frac{3 C_{20}}{2 r_{0}^{5}}+\frac{9 C_{22}}{r_{0}^{5}}=0, & \text { for SEP. }\end{cases}
$$

The crosses in Figure 1 indicate the four EPs of the example.

Denoting the deviations from the EPs as $\rho=(\xi, \eta, \zeta)$ and linearizing Equation (4) around the EPs, we have

$$
\left\{\begin{array}{l}
\ddot{\xi}-2 \dot{\eta}-\left(1+V_{x x}^{0}\right) \xi=0 \\
\ddot{\eta}+2 \dot{\xi}-\left(1+V_{y y}^{0}\right) \eta=0 \\
\ddot{\zeta}-V_{z z}^{0} \zeta=0=0
\end{array}\right.
$$

where $V_{* *}^{0}$ is the second derivative of $V$ with respect to the coordinates taking values at the EPs. Obviously, the planar motion is decoupled from the vertical motion in the linearized model of Equation (11). For both the LEPs and the SEPs, $V_{z z}^{0}<0$, and thus motion along the $z$-axis is a simple oscillation with the basic frequency as $\nu=\sqrt{-V_{z z}^{0}}$. For the planar motion, denoting the eigenvalues as $\lambda$, they satisfy

$$
\lambda^{4}+\left(2-V_{x x}^{0}-V_{y y}^{0}\right) \lambda^{2}+\left(1+V_{x x}^{0}\right)\left(1+V_{y y}^{0}\right)=0 .
$$

For the LEPs, the eigenvalues are of the following form:

$$
\lambda_{1,2}= \pm s, \quad \lambda_{3,4}= \pm i \omega,
$$

which means that the LEPs are unstable. The solution to Equation (11) can be written as

$$
\left\{\begin{array}{l}
\xi=C_{1} e^{s t}+C_{2} e^{-s t}+\alpha \cos \left(\omega t+\phi_{1}\right) \\
\eta=k_{1} C_{1} e^{s t}+k_{2} C_{2} e^{-s t}+k_{3} \alpha \sin \left(\omega t+\phi_{1}\right), \\
\zeta=\beta \cos \left(v t+\phi_{3}\right)
\end{array}\right.
$$

where $C_{1}, C_{2}, \alpha, \beta, \phi_{1}, \phi_{3}$ are constants that are determined by the initial conditions, and

$$
\begin{aligned}
& k_{1}=\frac{s^{2}-1-V_{x x}^{0}}{2 s}, k_{2}=\frac{1+V_{x x}^{0}-s^{2}}{2 s}, \\
& k_{3}=-\frac{1+V_{x x}^{0}+\omega^{2}}{2 \omega} .
\end{aligned}
$$

If we choose the initial conditions such that $C_{1}=C_{2}=0$, we get conditionally stable orbits around the LEPs. Then, $\alpha$ and $\beta$ can be taken as the size parameters of the in-plane and out-ofplane motions, respectively.

For the SEPs, the case is a little bit tricky. The right panel of Figure 1 shows how the stability changes with respect to the $C_{20}$ and $C_{22}$ values. Judging from the figure, for small values of $C_{20}$ and $C_{22}$ (i.e., values below the solid line in Figure 1), the eigenvalues are of the following form $\left(\omega_{l}<\omega_{s}\right)$ :

$$
\lambda_{1,2}= \pm i \omega_{l}, \quad \lambda_{3,4}= \pm i \omega_{s}
$$

which means that the motions are stable. The solution to Equation (11) is

$$
\left\{\begin{array}{l}
\xi=\alpha_{l} \cos \left(\omega_{l} t+\phi_{1}\right)+\alpha_{s} \cos \left(\omega_{s} t+\phi_{2}\right) \\
\eta=k_{l} \alpha_{l} \sin \left(\omega_{l} t+\phi_{1}\right)+k_{s} \alpha_{s} \sin \left(\omega_{s} t+\phi_{2}\right), \\
\zeta=\beta \cos \left(v t+\phi_{3}\right)
\end{array}\right.
$$

where $\alpha_{l}, \alpha_{s}, \beta, \phi_{1}, \phi_{2}, \phi_{3}$ are constants that are determined by the initial conditions;

$$
k_{l}=-\frac{1+V_{x x}^{0}+\omega_{l}^{2}}{2 \omega_{l}}, k_{s}=-\frac{1+V_{x x}^{0}+\omega_{s}^{2}}{2 \omega_{s}},
$$

$\alpha_{l}$, and $\alpha_{s}$ can be taken as the size parameter of each component of the planar motion; and $\beta$ can be taken the size parameter of the out-of-plane motion. Similar to the CRTBP, we call the components of the planar motion in Equation (13) the longperiod (with the basic frequency $\omega_{l}$ ) component and the shortperiod (with the basic frequency $\omega_{s}$ ) component. For specific combinations of $C_{20}$ and $C_{22}$ (i.e., values on the curve in the right panel of Figure 1), $\omega_{l}=\omega_{s}$ and

$$
\lambda_{1,2}=\lambda_{3,4}= \pm i \omega_{s}= \pm i \omega_{l} .
$$

For larger $C_{20}$ and $C_{22}$ terms (i.e., values above the curve in the right panel of Figure 1), the eigenvalues become

$$
\lambda_{1,2}=\gamma \pm i \omega, \quad \lambda_{3,4}=-\gamma \pm i \omega,
$$

and the motions become spirally unstable. The stabilitychanging process with the increase of $C_{20}$ and $C_{22}$ described above are discussed in Scheeres (1994). This process is similar to the stability change process around the triangular libration points of the CRTBP with the increase of the mass parameter $\mu$ (Szebehely 1967).

\subsection{Periodic Orbits}

According to the Lyapunov theorem, there are two families of POs for the LEPs: the planar family and the vertical family, with the orbital period approaching $2 \pi / \omega$ and $2 \pi / v$, respectively, as their orbital sizes approach zero. For the SEPs, there are three families of POs. Two of them are restricted within the $x-y$ plane: the long-period family and the shortperiod family. Their orbital periods approach $2 \pi / \omega_{l}$ and 

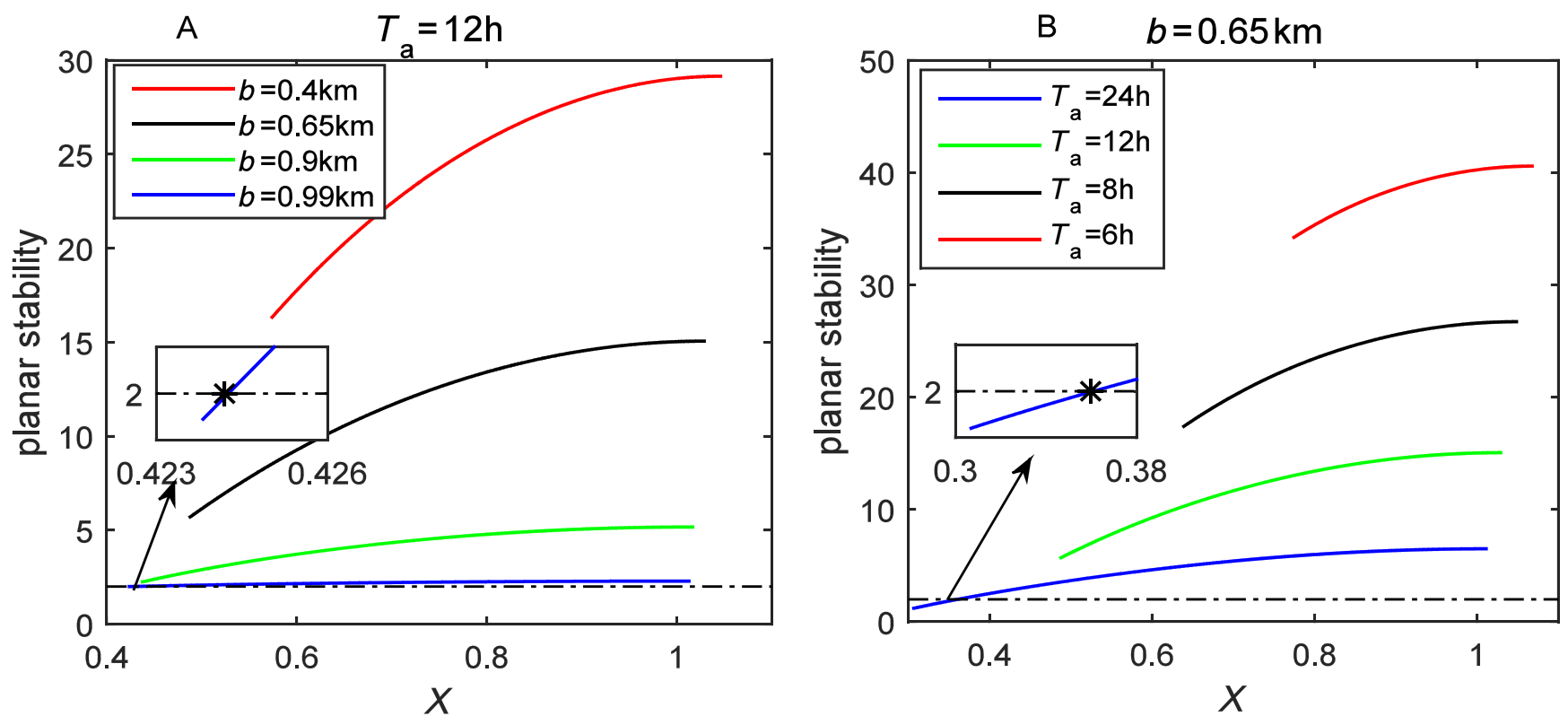

Figure 3. Planar stability curves of the planar Lyapunov families around the LEP for asteroids with different shapes (left) and rotations (right). The dashed horizontal lines indicate the critical value of 2 for the planar stability parameter; at values larger than this, the periodic orbit becomes unstable.

$2 \pi / \omega_{s}$, respectively, as the orbital size approaches zero. The third family is a spatial periodic family, namely, the vertical Lyapunov family, with an orbital period approaching $2 \pi / v$ as the orbital size approaches zero.

Denote the state vector of the system as $X=(x, y, z, \dot{x}, \dot{y}, \dot{z})$ and the trajectory of Equation (4) with an initial value $X_{0}=\left(x_{0}, y_{0}, z_{0}, \dot{x_{0}}, \dot{y}_{0}, \dot{z}_{0}\right)$ as $X(t)=\left(X_{0}, t\right)$. Periodic orbits with the orbital period $T$ satisfy

$$
X\left(X_{0}, T\right)-X_{0}=0 .
$$

Due to the symmetry of the POs, Equation (15) can be reduced to simpler forms. The planar and vertical Lyapunov orbits around the LEPs are symmetric with respect to the $x-z$ plane. As a result, if the initial value is chosen as the perpendicular intersection point of the orbit with the $x-z$ plane, Equation (15) is reduced to

$$
\begin{aligned}
& y\left(x_{0}, z_{0}, \dot{y}_{0}, T / 2\right)=0, \dot{x}\left(x_{0}, z_{0}, \dot{y}_{0}, T / 2\right)=0, \\
& \dot{z}\left(x_{0}, z_{0}, \dot{y_{0}}, T / 2\right)=0 .
\end{aligned}
$$

The planar long- and short-period orbits and the vertical Lyapunov orbits around the SEPs are symmetric with respect to the $y-z$ plane. As a result, if the initial value is chosen as the perpendicular intersection point of the orbit with the $y-z$ plane, Equation (15) is reduced to

$$
\begin{aligned}
& x\left(x_{0}, z_{0}, \dot{y}_{0}, T / 2\right)=0, \dot{y}\left(x_{0}, z_{0}, \dot{y}_{0}, T / 2\right)=0, \\
& \dot{z}\left(x_{0}, z_{0}, \dot{y}_{0}, T / 2\right)=0 .
\end{aligned}
$$

For the planar POs, the third equation in Equations (16) and (17) and the variable $z_{0}$ are unnecessary. Details on the numerical approach to these periodic families are not given here. In this work, we use the well-known predictor-corrector algorithm (Press 2007). To start this algorithm, an initial guess for a small-size orbit is necessary, which can be provided by the linearized results in the above subsection.

One remark is made here. As already mentioned, when viewing in the inertial frame, the EPs are exact 1:1 resonance orbits. The POs around the EPs discussed in this section are also in the 1:1 resonance zone but not in exact 1:1 resonance with the asteroid's rotation; i.e., the orbits are periodic in the asteroid's body-fixed frame but not in the inertial frame. More specifically:

(1) The planar Lyapunov orbits around the LEPs are actually eccentric 1:1 resonance orbits, with the periapse and apoapse aligning along the asteroid's long axis. An increase in the size of the planar Lyapunov orbit can be interpreted as an increase in the orbital eccentricity in the inertial frame. The vertical Lyapunov orbits around the LEPs are actually inclined near-circular 1:1 resonance orbits with the ascending and descending nodes aligning along the asteroid's long axis. The increase in the size of the vertical Lyapunov orbit corresponds to the increase in the orbital inclination in the inertial frame.

(2) For the short-period orbits around the SEPs, the case is the same as that of the planar Lyapunov orbits around the LEPs, but the eccentric orbits have the periapse and apoapse aligning along the asteroid's short axis. The long-period orbits around the SEPs are actually planar near-circular orbits in the inertial frame with a longperiod difference in the semimajor axis from that of the exact 1:1 resonance orbit. The vertical Lyapunov orbits around the SEPs are the same as the vertical Lyapunov orbits around the LEPs but with the ascending and descending nodes aligning along the asteroid's short axis.

In the following, we will focus on these POs and study their stability properties with respect to their sizes. Based on the above argument, the study can also be taken as the study of 1:1 resonance orbits with different orbital eccentricities or inclinations. Also, we will study the invariant manifolds associated with the LEPs.

\section{Dynamics Around the LEPs}

\subsection{The Planar Lyapunov Family}

As mentioned above, there is a planar periodic family generated from the LEP that we call the planar Lyapunov family. Taking the asteroid with $a: b: c=1: 0.65: 0.4 \mathrm{~km}$ and a 


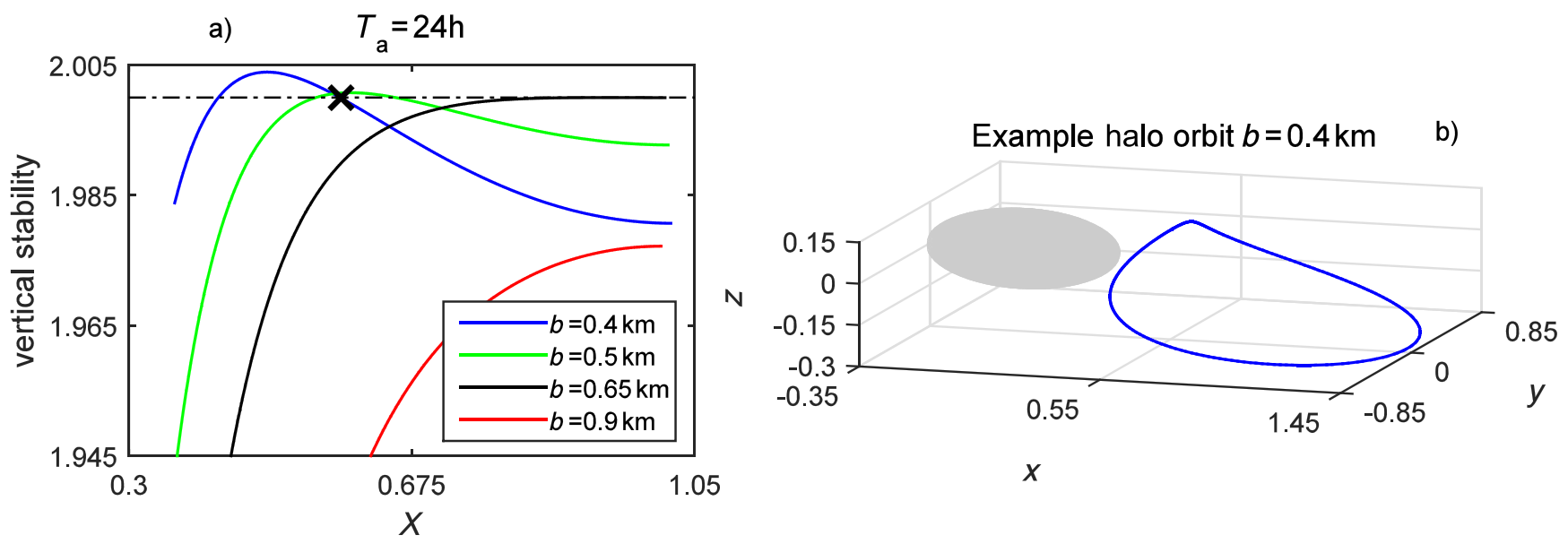

Figure 4. Vertical stability curve of a planar family for a specific asteroid and an example halo orbit.
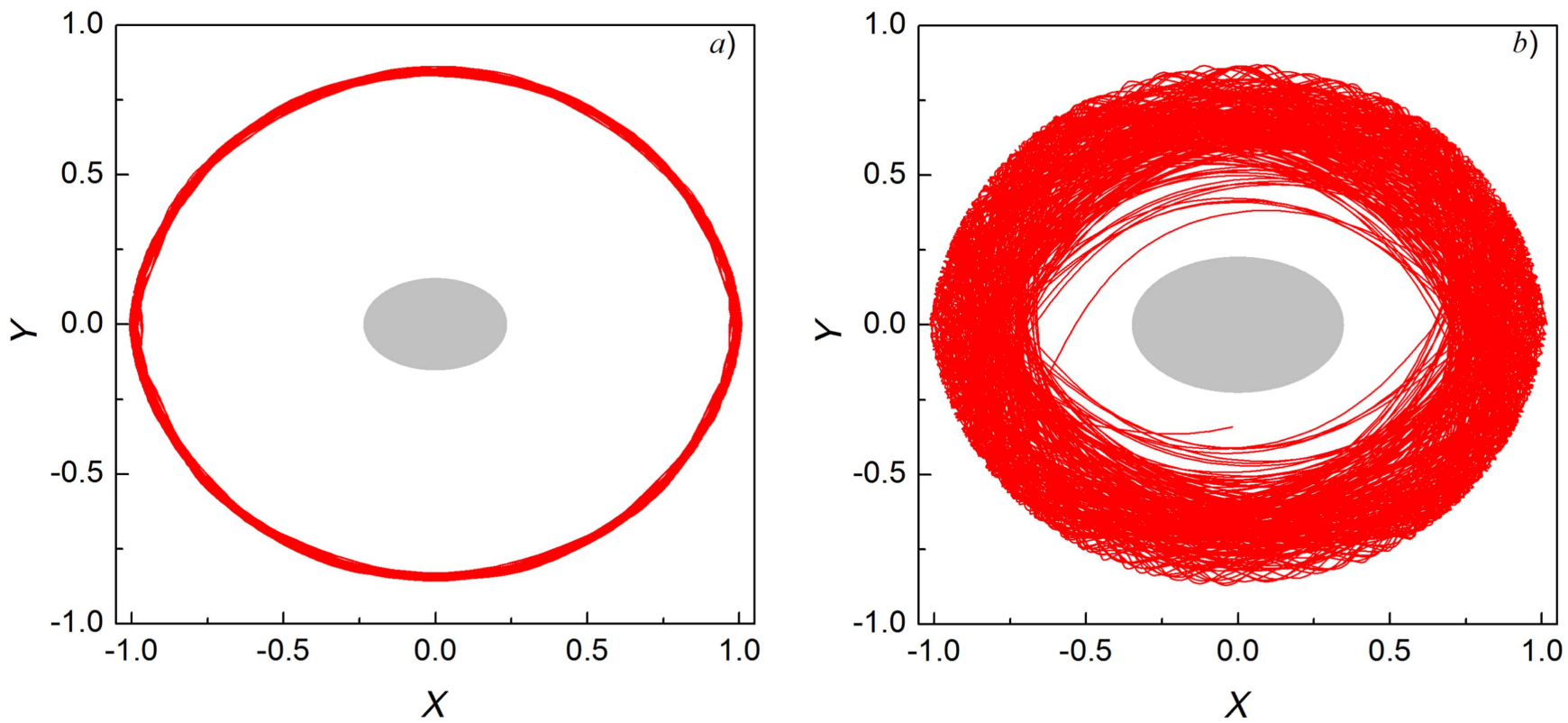

Figure 5. Inward branch of the unstable invariant manifolds associated with the right LEP. Left: the rotation period of the asteroid is $36 \mathrm{hr}$. The integration time is 1000 rotation periods of the asteroid. Right: the rotation period of the asteroid is $20 \mathrm{hr}$. The integration is stopped when the manifold intersects the asteroid's Brillouin sphere.

rotation period of $12 \mathrm{hr}$ as an example, Figure 2 shows some examples of this family. These orbits are actually in 1:1 resonance with the asteroid's rotation in the inertial frame, with the periapse and apoapse aligning along the asteroid's long axis in its body-fixed frame.

To observe the effects of the asteroid's shape and rotation on the planar family, two sets of simulations are performed. For both sets, the semiaxes $a$ and $c$ are fixed at values of 1 and $0.4 \mathrm{~km}$. The results are given in Figure 3 . The abscissa $X$ is the $x$-coordinate of the left perpendicular intersection point of the planar orbits with the $x$-axis (black stars in Figure 2). For the first simulation, the asteroid's rotation period is fixed at $12 \mathrm{hr}$, and the semiaxis $b$ is varied from 0.4 to $0.99 \mathrm{~km}$. Given each combination of these physical parameters, first the EP is found, and then the POs around it are numerically obtained and continued until the PO intersects with the asteroid, for a practical consideration. The planar stability curves are given in Figure 3 (a). In our work, we use the stability parameters given by Howell \& Spencer (1986). An obvious fact from this figure is that the instability of this family becomes stronger with decreasing $b$, i.e., a larger $C_{22}$ term. For the second simulation, the asteroid's shape is fixed at $b=0.65 \mathrm{~km}$, while its rotation period (denoted as $T_{\mathrm{a}}$ in Figure 3(b)) is changed from 4 to $24 \mathrm{hr}$. From this figure, it can be seen that the instability of this family also becomes stronger when the asteroid rotates faster. This similarity is understandable. As mentioned in Section 2.1, increasing the asteroid's rotation speed is equivalent to elongating its shape. As a result, in the following studies, we change the 2OD gravitational potential by varying either the asteroid's shape or its rotation speed, but not both.

Additionally, for small $C_{22}$ values (Figure 3), there exists a small stable region in which the POs are extremely close to the asteroid's surface, namely, stable orbits with very large amplitudes. This indicates that highly eccentric stable 1:1 resonance orbits can exist for a $20 D$ gravitational potential with small $C_{22}$ values. Since parts of these orbits are close to the asteroid's surface, even when we are dealing with small $C_{22}$ values, the higher-order nonspherical terms that we neglect may also play their roles. It would be interesting to study the effects of these higher-order nonspherical terms for specific 

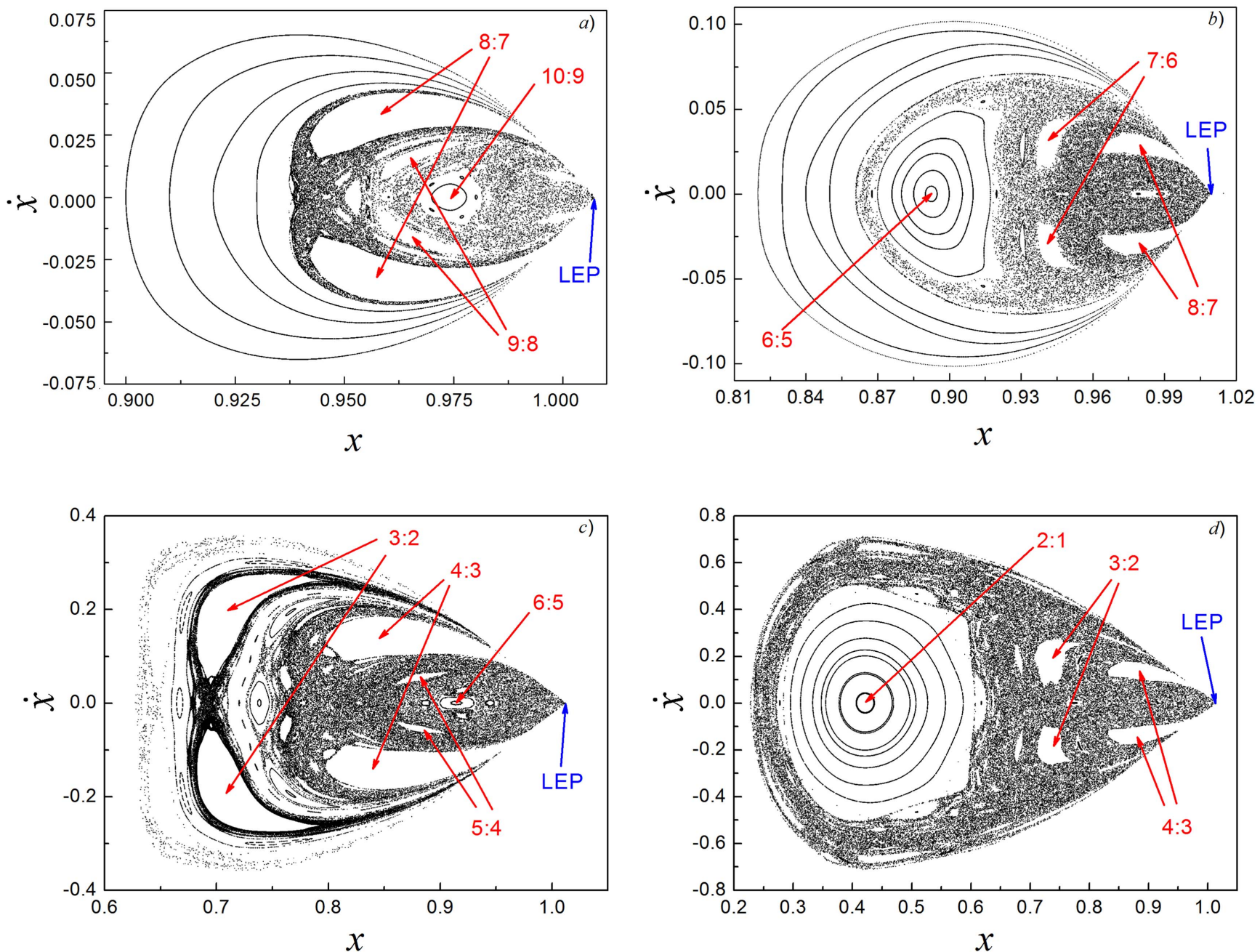

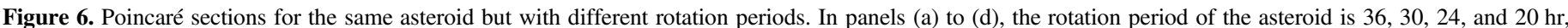

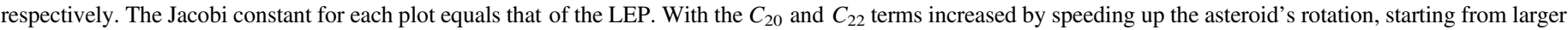

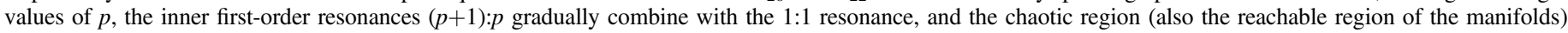
gradually approaches the asteroid.

asteroids and observe their effects on the persistence or disappearance of these stable orbits.

For the collinear libration points in the CRTBP, the wellknown halo family bifurcates from a critical member of the planar Lyapunov family generated from the collinear libration points. Since the LEPs in this work are dynamically equivalent to the collinear libration points in the sense that they are both of the saddle $\times$ center $\times$ center type (Gómez et al. 2004), a similar phenomenon is expected. Figure 4(a) shows the vertical stability curve of the planar Lyapunov family. The rotation period of the asteroid is fixed at $24 \mathrm{hr}$. The semiaxes $a$ and $c$ are fixed at 1 and $0.4 \mathrm{~km}$, while the semiaxis $b$ is varied. From this figure, we know that the vertical critical orbit can only exist for highly elongated or fast-rotating asteroids. As a result, the spatial periodic family (which we also call the halo family) can only exist for asteroids with large 2OD terms. Figure 4(b) shows an example halo orbit, corresponding to the one denoted by a cross in Figure 4(a). Again, these spatial periodic orbits are generally of large amplitudes, with parts of them very close to the asteroid's surface, and the higher-order nonspherical terms that we neglect may also play their roles. In this case, their effects may be stronger than in the case of Figure 3, because the higher-order nonspherical terms are usually larger if the asteroid has larger 2OD terms. Anyway, it is interesting to study the effects of these higher-order nonspherical terms for specific asteroids and observe the persistence or disappearance of these spatial orbits with their effects.

\subsection{Invariant Manifolds}

As pointed out in Section 2, the LEPs are generally unstable. Since a pair of real eigenvalues exists for the LEPs, there are stable and unstable invariant manifolds associated with them, exactly the same as the collinear libration points of the CRTBP. Taking the asteroid with a shape of $a: b: c=$ 1:0.65:0.4 km as an example, Figure 5 shows the inward branch of the unstable invariant manifolds of the right LEP for a rotation period of $36 \mathrm{hr}$ (a) and $20 \mathrm{hr}$ (b). The invariant manifolds can enter the asteroid's Brillouin sphere in Figure 5(b) but cannot in Figure 5(a). The reason we stop our integration at the Brillouin sphere instead of the asteroid's surface is that the gravitational potential field given by the spherical harmonics usually diverges within this sphere. 

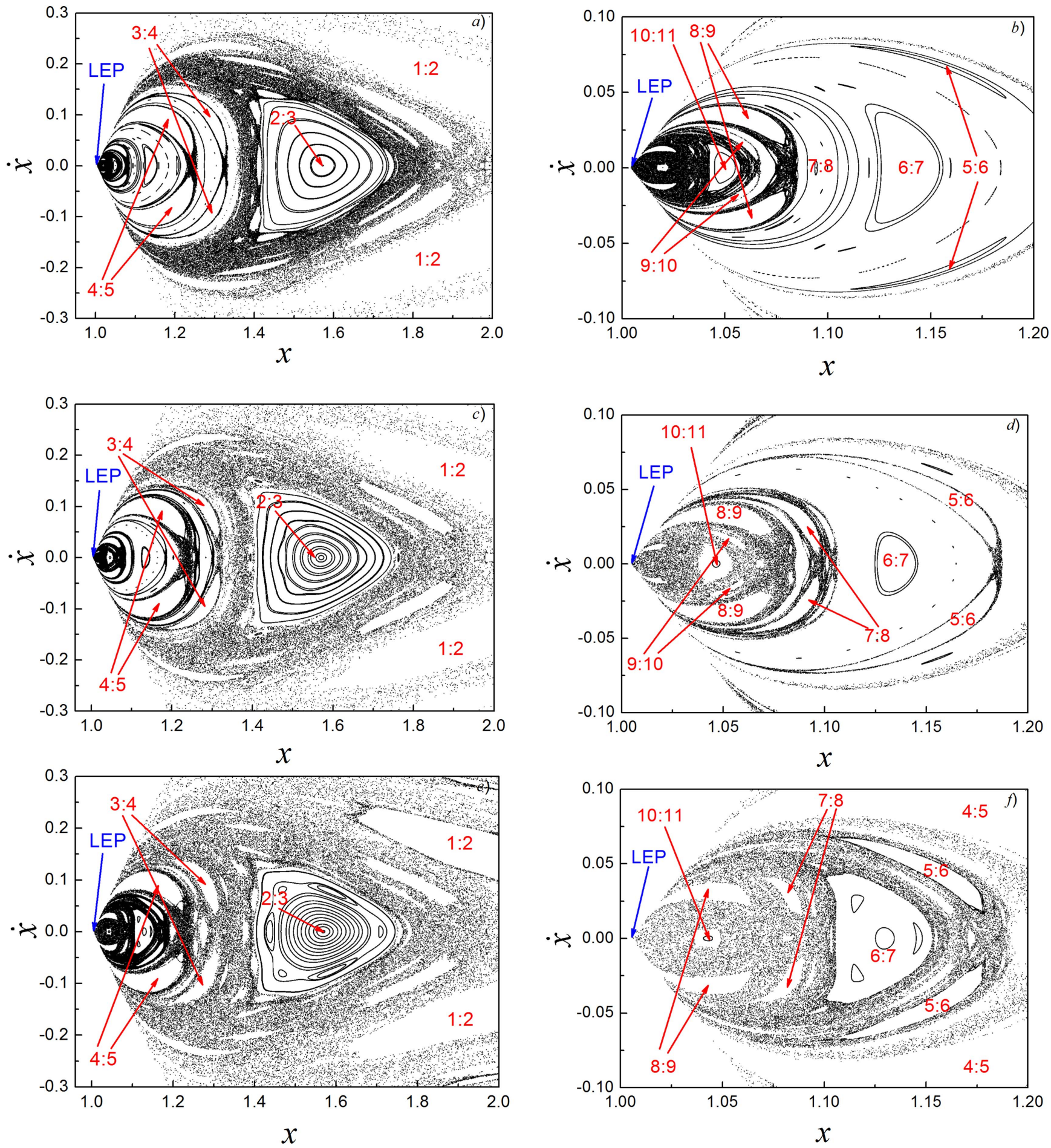

Figure 7. Same as Figure 6 but for the outward branch of the invariant manifold associated with the LEP.

The reason that the invariant manifolds in Figure 5(a) cannot approach the asteroid is explained as follows. Figure 6 shows the Poincaré maps with a Jacobi constant equaling that of the LEP. The map is generated every time the orbits intersect the positive $x$-axis. We record the point of the orbit every time it intersects the $x$-axis. The abscissa in the maps is the $x$-coordinate of the intersection point, and the ordinate is the corresponding $\dot{x}$. We only record the intersection point with $\dot{y} \geqslant 0$. The asteroid's shape is the same as that in Figure 5. The rotation period of the asteroid, however, is varied, taking values of $36,30,24$, and $20 \mathrm{hr}$ in panels (a) to (d), respectively. In each panel of Figure 6 , the rightmost point on the tip of the tongue is the LEP, denoted as "LEP." The chaotic region containing the LEP can be taken as the reachable region of the inward branch of the invariant manifolds. Some resonances are denoted in these maps. For a resonance $p: q$, the asteroid rotates $q$ times while the particle circles $p$ orbits around the asteroid in the inertial frame. Here, $|q-p|$ is called the resonance order. Usually, the strength of higher-order resonances is weak (Kaula 1966; Murray \& Dermott 1999). When $q<p(q>p)$, 


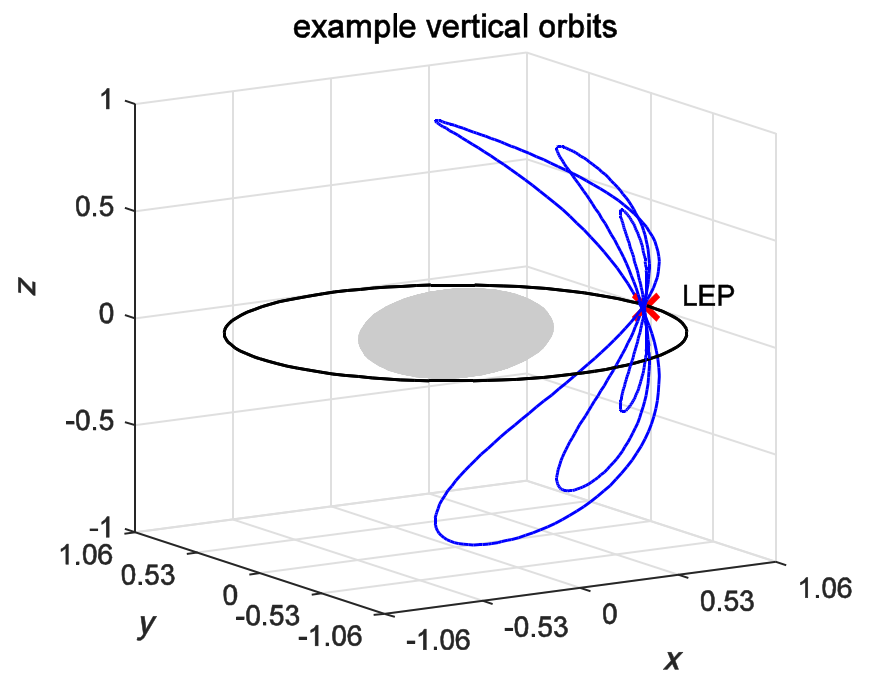

Figure 8. Example orbits of the vertical Lyapunov family.

the resonance is inner (outer) resonance. Only first-order resonances are marked in Figure 6, since they usually dominate over higher-order resonances.

The resonance overlap process and the accessible region of the inward manifolds in Figure 6 are described as follows. (1) In Figure 6(a), where the 1:1 resonance orbit is far away from the asteroid and the 2OD terms are small, the 8:7, 9:8, and 10:9 resonances overlap with each other. However, there are regular Kolmogorov-Arnold-Moser (KAM) curves (Siegel \& Moser 1971) between the 8:7 and 7:6 resonances, preventing the invariant manifolds from migrating further inward. (2) In Figure 6(b), the 6:5 resonance overlaps with nearby first-order resonances, allowing the invariant manifolds to access regions closer to the asteroid than that of Figure 6(a). Nevertheless, the regular KAM curves between the 6:5 and 5:4 resonances prevents the invariant manifolds from reaching regions interior to the 5:4 resonance. (3) Similarly, the thin area of regular KAM curves between the 4:3 and 3:2 resonances in Figure 6(c) prevents the invariant manifolds from reaching regions interior of the 3:2 resonance. (4) Finally, the 2:1 resonance overlaps with nearby resonances in Figure 6(d), and the invariant manifolds can reach regions interior of this resonance, which in this case already touches the asteroid's surface. Figure 6 shows us the following information: starting from large $p$ values, the first-order resonances $(p+1): p$ overlap with the 1:1 resonance. With the 2OD terms increasing from larger to smaller values of $p$, more first-order resonances overlap each other, allowing the invariant manifolds from the $1: 1$ resonance to reach the inner regions until they reach the asteroid's surface.

For the outward branch of the invariant manifolds associated with the LEPs, a similar phenomenon happens. Figure 7 gives the Poincare maps. We record the point of the orbit every time it intersects the $x$-axis, but for the point with $\dot{y} \leqslant 0$. The asteroid's shape is the same as that in Figure 6 . The rotation period of the asteroid, however, is different, taking values of $56 \mathrm{hr}$ for panels (a) and (b), $52 \mathrm{hr}$ for panels (c) and (d), and $48 \mathrm{hr}$ for panels (e) and (f). Similar to Figure 6, the chaotic region containing the LEP can be taken as the accessible region of the outward branch of the invariant manifolds. The right panels of Figure 7 are local magnifications of the left panels in the proximity of the LEP. When the asteroid rotates with a period of $56 \mathrm{hr}$, the 2OD terms are small. (1) In Figure 7(b), the outward branch of the invariant manifolds is "blocked" by the
KAM curves between the 8:9 and 9:10 resonances. This means that the outward branch of the invariant manifold of the LEP cannot go outward beyond the region where the 8:9 resonance is. In Figure 7(a), the 2:3 resonance is separated from the 3:4 resonance by KAM curves. (2) In Figure 7(d), the chaotic region containing the LEP incorporates the 8:9 resonance but is blocked by KAM curves between the $8: 9$ and 7:8 resonances. In Figure 7(c), the chaotic region containing the 1:2 and $2: 3$ resonances now incorporates the 3:4 resonance but is still separated from the chaotic region containing the LEP. (3) In Figure 7(f), the chaotic region containing the LEP incorporates the 4:5 resonance. Meanwhile, from Figure 7(e), we know that the chaotic region containing the $1: 2,2: 3$, and $3: 4$ resonances also incorporates the $4: 5$ resonance. This means that the two chaotic regions finally "meet" with each other in the phase space, indicating that the outward branch of the invariant manifold can go beyond the 1:2 resonance and even further away from the asteroid. Actually, the chaotic region containing the 1:2 resonance extends to infinity, so there is a possibility for particles starting from the LEP to escape from the asteroid's gravitational potential.

The scenarios described in Figures 6 and 7 are the same as those of the invariant manifolds associated with the collinear libration points of the CRTBP (Conley 1968; Llibre et al. 1985; Koon et al. 2000).

Also similar to the collinear libration point of the CRTBP, there are invariant manifolds associated with the planar Lyapunov orbits around the LEPs in the above subsection. They and the invariant manifolds associated with the LEP described above can be used for probes to land on or ascend from the asteroids (Herrera-Sucarrat 2014). Similar to missions around the collinear libration points in the CRTBP (Howell et al. 1997; Sweeter et al. 2011), heteroclinic orbits can be used for probes to travel between the LEPs and SEPs.

\subsection{The Vertical Lyapunov Family}

Given $a: b: c=1: 0.65: 0.4 \mathrm{~km}$ and the rotation period of the asteroid at $12 \mathrm{hr}$, Figure 8 shows some examples of members of the vertical Lyapunov family in the rotation frame with increasing orbital amplitude, which is defined as the z-coordinate of the perpendicular intersection point of the orbits with the $x-z$ plane. The black orbit is the stable retrograde orbit in the equatorial plane on which the vertical family ends. In the inertial frame, these orbits are actually inclined near-circular 1:1 spin-orbit resonance orbits, with their ascending or descending nodes along the asteroid's long axis.

Figure 9 shows the $Z-C$ curve (left) and $T-C$ curve (right) of this family for different asteroids. The shape of the asteroid is fixed as $a: b: c=1: 0.65: 0.4 \mathrm{~km}$. When the asteroid rotates slowly, the genealogy of the family can be described as follows. Starting from the LEP, the $z$-coordinate of the orbits first increases (meanwhile, in the inertial frame, the orbital inclination of the 1:1 spin-orbit resonance orbit increases) to a critical value (around 1, corresponding to a polar orbit in the inertial frame) and then decreases to zero (meanwhile, in the inertial frame, the orbital inclination of the 1:1 spin-orbit resonance orbits further increases from $90^{\circ}$ to $180^{\circ}$ ). When the asteroid rotates faster, the $Z-C$ curve is obviously distorted (such as the one for $T_{\mathrm{a}}=4 \mathrm{hr}$ in Figure 9(a)) and finally breaks up and combines with another period family (denoted as dashed curves in Figure 9) to form two new families (two black lines for $T_{\mathrm{a}}=3.4 \mathrm{hr}$ ). Additionally, these orbits are generally 
a)

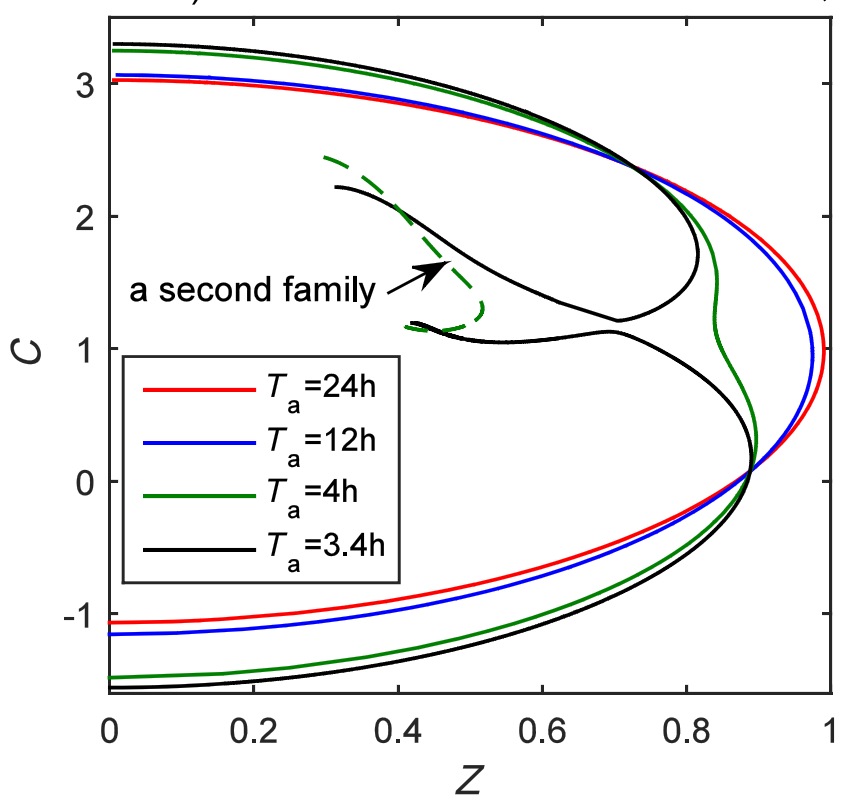

b)

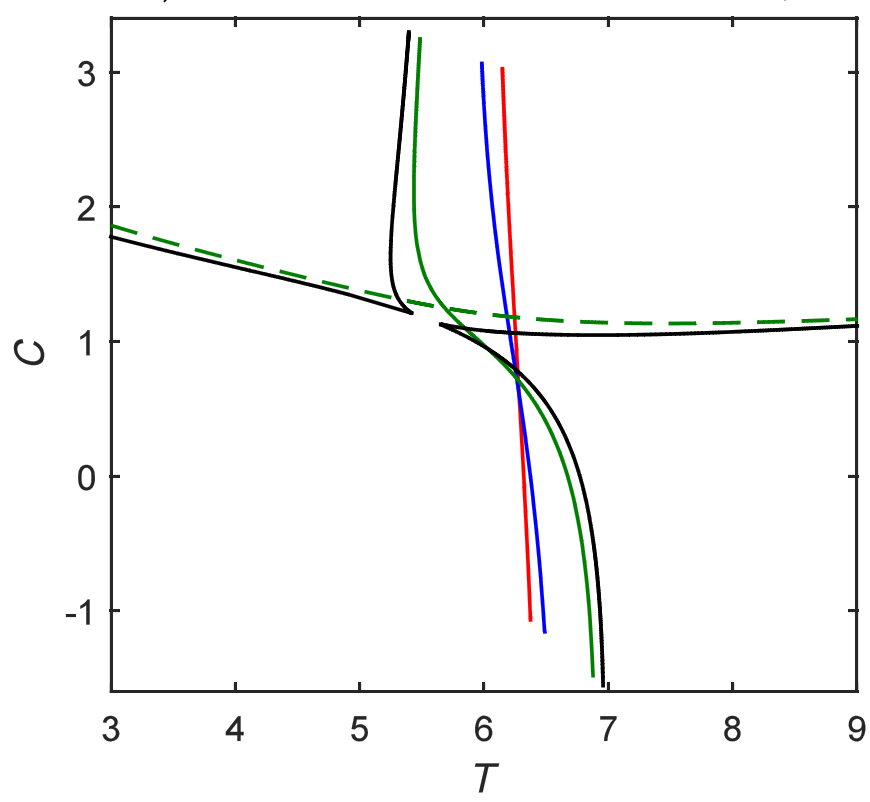

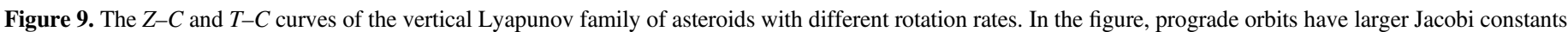
than retrograde orbits.

unstable, indicating that the $1: 1$ spin-orbit resonance orbits with ascending or descending nodes along the asteroid's long axis are generally unstable.

\section{Dynamics Around the SEPs}

As mentioned in Section 2.2, with the 2OD terms increasing, the SEPs change from stable to spirally unstable. The asteroid's shape is $a=1 \mathrm{~km}, b=0.6 \mathrm{~km}$, and $c=0.4 \mathrm{~km}$. Assuming its rotation period is $12 \mathrm{hr}$, we have

$$
C_{20}^{0}=-2.615478 \times 10^{-2}, \quad C_{22}^{0}=8.047625 \times 10^{-3} .
$$

The true values of $C_{20}$ and $C_{22}$ are taken as

$$
C_{20}=\epsilon C_{20}^{0}, C_{22}=\epsilon C_{22}^{0},
$$

where $\epsilon$ is a parameter. Keeping the asteroid's shape unchanged, according to Equation (9), it is easy to know that the true rotation period of the asteroid is

$$
T_{a}=12 h / \epsilon^{3 / 4} \text {. }
$$

Obviously, the larger $\epsilon$ is, the faster the asteroid rotates. Our studies find that there is a critical value $\epsilon_{c}$ around 0.80 . When $\epsilon<\epsilon_{c}$, the SEPs are stable, and when $\epsilon>\epsilon_{c}$, the SEPs are unstable. Here, we interpret changes of the parameter $\epsilon$ as changes in the asteroid's rotation speed, but, according to the arguments in Section 2.1, we can also interpret them as changes in the asteroid's shape.

\subsection{The Short-period Family}

If the SEPs are stable, according to Section 2, there are two planar Lyapunov families: the short-period family and the long-period family. Similarly, given $a: b: c=1: 0.65: 0.4 \mathrm{~km}$ and the rotation period of the asteroid at $12 \mathrm{hr}$, the left panel of Figure 10 gives some examples of these two families. The long-period orbits are generally more elongated than the short-period ones. As mentioned in Section 2.3, the shortperiod orbits are eccentric 1:1 resonance orbits in the inertial frame with the periapse and apoapse aligning along the asteroid's short axis, and the long-period orbits are nearcircular 1:1 resonance orbits in the inertial frame with a longperiod variation of the semimajor axis. In this section, the short-period family is studied first.

The right panel of Figure 10 shows the stability curve of the short-period family for different values of $\epsilon$ when the SEP is stable. The abscissa is the $y$-coordinate of the lower perpendicular intersection point between the periodic orbit and the $y$-axis, also for subsequent figures in this paper. From smaller to larger orbits (i.e., from larger to smaller values of $Y$ ), the family is continued until the orbit collides with the asteroid's surface. It can be seen that most members of the short-period family are stable, but a small portion of it (with a very large amplitude) is unstable. Moreover, with an increase of the nonspherical terms (here interpreted as an increase in the parameter $\epsilon$ ), there is a tendency for more members to become unstable due to the stronger perturbations. Interpreting these results in the inertial frame, the 1:1 spin-orbit resonance orbits with the periapse and apoapse aligning along the asteroid's short axis are stable, except for the ones with a very large orbital eccentricity.

\subsection{The Long-period Family}

Taking $\epsilon=0.3$ in Figure 10 as an example, Figure 11 shows the $T-C$ curves of the long-period family. It is worth noting that this family terminates onto a short-period orbit traveling four times. From this short-period orbit, another periodic family (which we denote as $B(4,5)$ in Figure 11 ) bifurcates, connecting it with a short-period orbit traveling five times. From this shortperiod orbit, yet another periodic family (which we denote as $B(5,6)$ in Figure 10) bifurcates, connecting it with a short-period orbit traveling six times. This genealogy of periodic families is exactly the same as that between the long-and short-period families around the triangular libration points in the CRTBP 

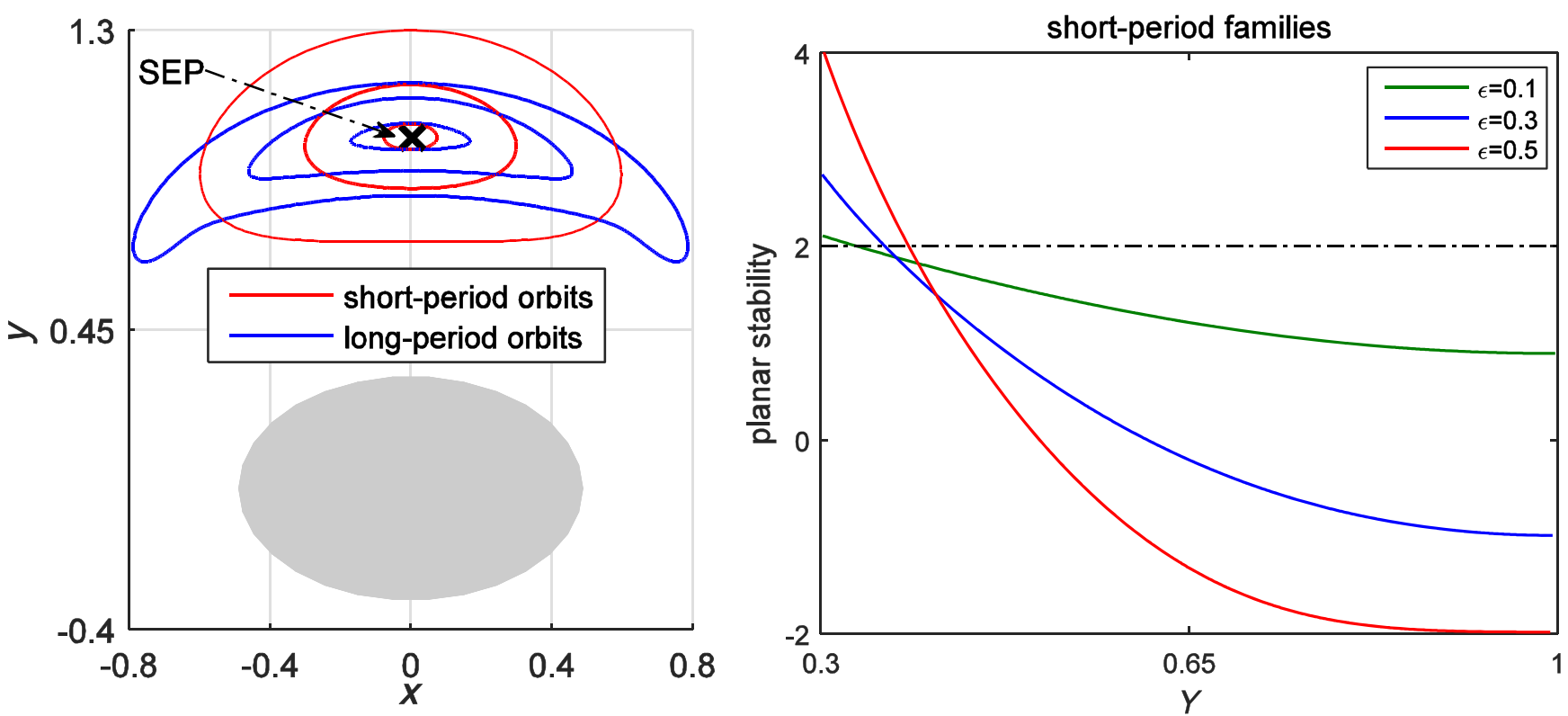

Figure 10. Left: example orbits of the long- and short-period families around the SEP. Right: planar stability curves of the short-period family around the SEP before the orbits collide with the asteroid. Different curves correspond to different values of $\epsilon$.
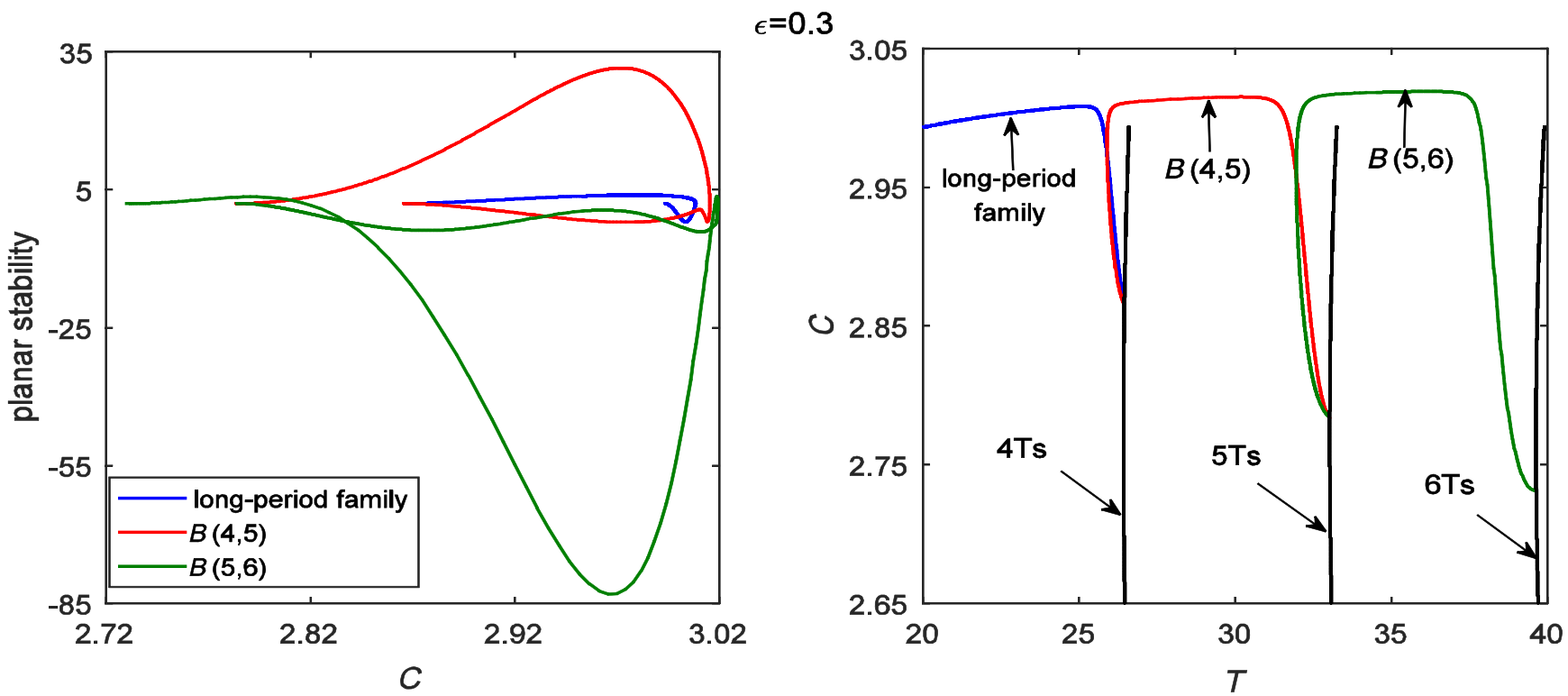

Figure 11. Planar stability curve (left) and $T-C$ curve (right) of the long-period family, along with the curves for the families $B(4,5)$ and $B(5,6)$. In the right frame, the near-vertical black lines indicate the $T-C$ curves of the short-period family but with the period multiplied four, five, and six times. In the figure, "Ts" indicates the period of the short-period family.

(Hou \& Liu 2009). More specifically, if the ratio between the two basic frequencies in Equation (14) satisfies $\omega_{s} / \omega_{l} \in(N-1$, $N)$, the long-period family terminates on an $N$-bifurcation shortperiod orbit. ${ }^{3}$ Starting from this $N$-bifurcation short-period orbit, there are a series of periodic families $B(N+K, N+K+1)$, where $k \geqslant 0$ connecting an $(N+K)$-bifurcation short-period orbit with an $(N+K+1)$-bifurcation short-period orbit. Additionally, from numerical simulations, it is found that this genealogy holds for asteroids with different $a: b: c$ values and rotation periods.

\footnotetext{
3 An $N$-bifurcation orbit is not a bifurcation orbit itself but becomes one when it travels $N$ times (Henrard 2002).
}

One remark is made here. When computing these periodic families, we may come across some periodic orbits that collide with the asteroid. In order to show the genealogy of these periodic families in the $2 \mathrm{OD}$ gravitational potential, we neglect the physical size of the asteroid when producing Figure 11. Additionally, it would be interesting to study the persistence of the genealogy between these families with higher-order nonspherical perturbations for specific asteroids.

With the parameter $\epsilon$ increasing, the two basic frequencies $\omega_{l}$ and $\omega_{s}$ approach each other, and $N$ approaches 2 . When $\epsilon$ grows from values smaller than to larger than $\epsilon_{c}$, the SEP becomes unstable. In the following, we show how the family genealogy changes when the SEPs turn from stable to unstable. Studies 

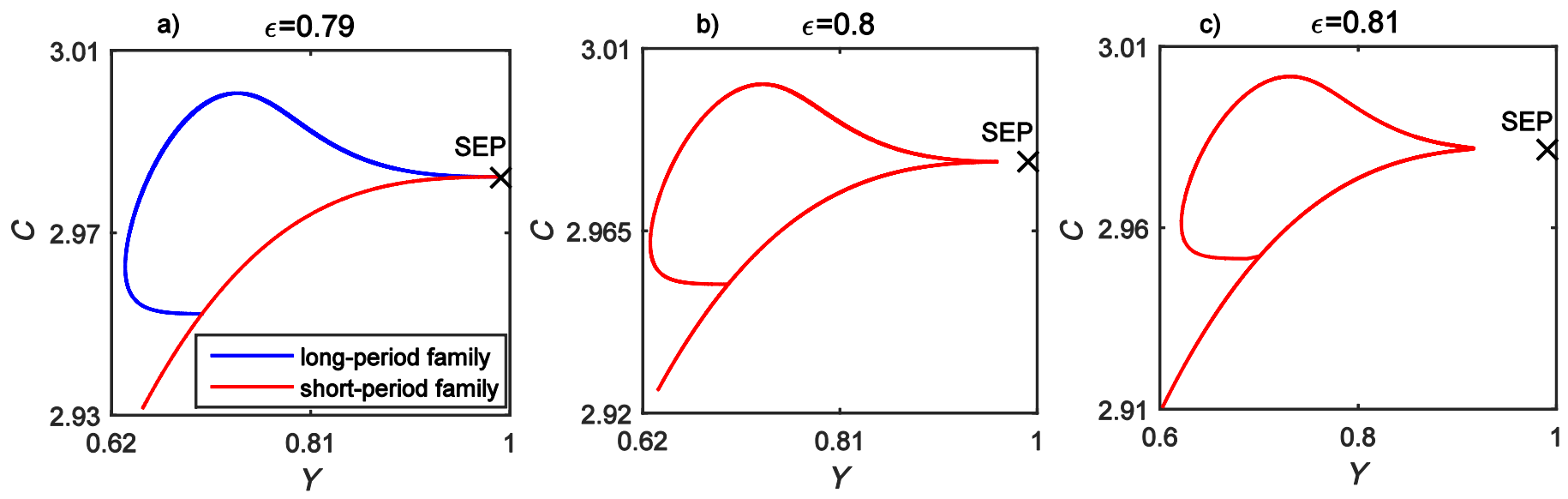

Figure 12. The $Y-C$ curves of the long- and short-period families for $\epsilon=0.79,0.80$, and 0.81 .
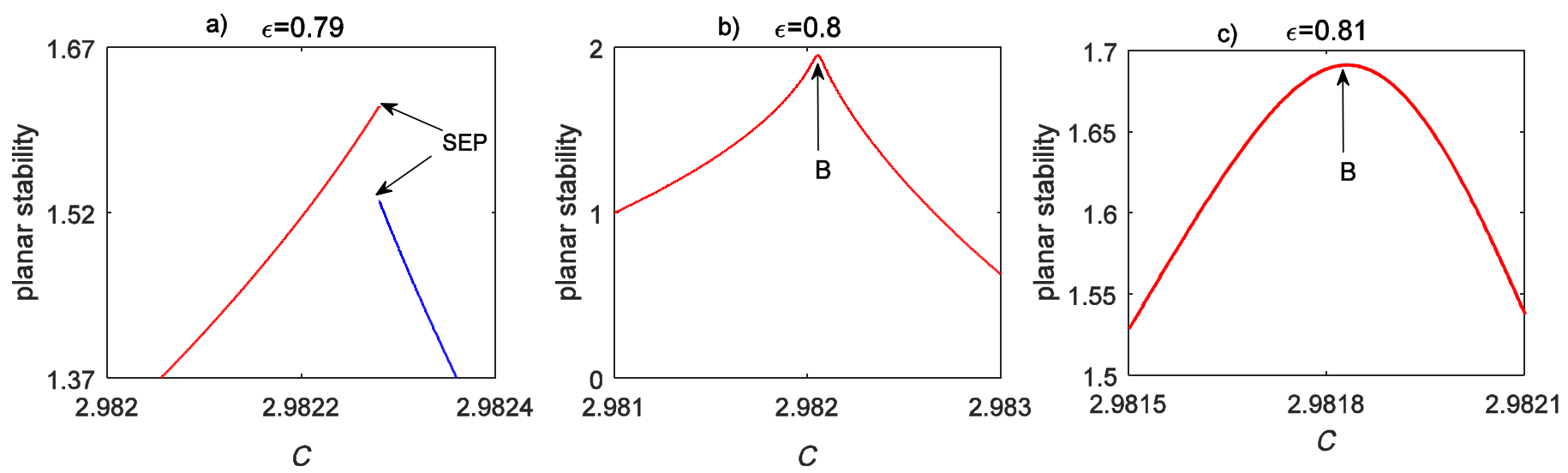

Figure 13. Local details of the planar stability curve of the periodic families in the proximity of the SEP. In the left frame, the long-and short-period families are separate. In the middle and right frames, the two combine to form a single periodic family.

show that when $\epsilon$ is very close to but smaller than $\epsilon_{c}$, the longand short-period families still separate from each other, and the former terminates on a two-bifurcation orbit of the latter, as shown by Figure 12(a) $(\epsilon=0.79)$. When $\epsilon=\epsilon_{c}$, the two families join each other at the SEP. When $\epsilon$ increases to slightly larger than $\epsilon_{c}$, the two families combine to form a single family and detach from the SEP, as shown by Figure 12(b) $(\epsilon=0.8)$. With $\epsilon$ further increasing, the distance of the family from the SEP in the $Y-C$ curve becomes larger, as shown by Figure 12(c) $(\epsilon=0.81)$. The whole process (i.e., the long- and short-period families joining together to form a single-period family and the SEP becoming unstable) is again exactly the same as that of the triangular libration point when the mass parameter $\mu$ increases from values smaller than the Routh's critical value to values larger than it (Hou \& Liu 2009).

Figure 13 shows the planar stability curves corresponding to Figure 11. In Figure 13(a), where $\epsilon<\epsilon_{c}$, the two families are separated. In the figure, "SEP" indicates that the two families are generated from infinitesimally small orbits around the SEP. With $\epsilon$ approaching $\epsilon_{c}$, the two families join together at the SEP, and their stability parameter equals 2 . With $\epsilon$ increasing to values larger than $\epsilon_{c}$, the two families join together and detach from the SEP, as shown in Figures 13(b) and (c). In this case, although the SEPs become unstable themselves, there exist stable POs of finite sizes around them. One example is given in Figure 14. The blue trajectory starting from the SEP (with a small perturbation of $10^{-2}$ on the $y$-axis) is unstable,

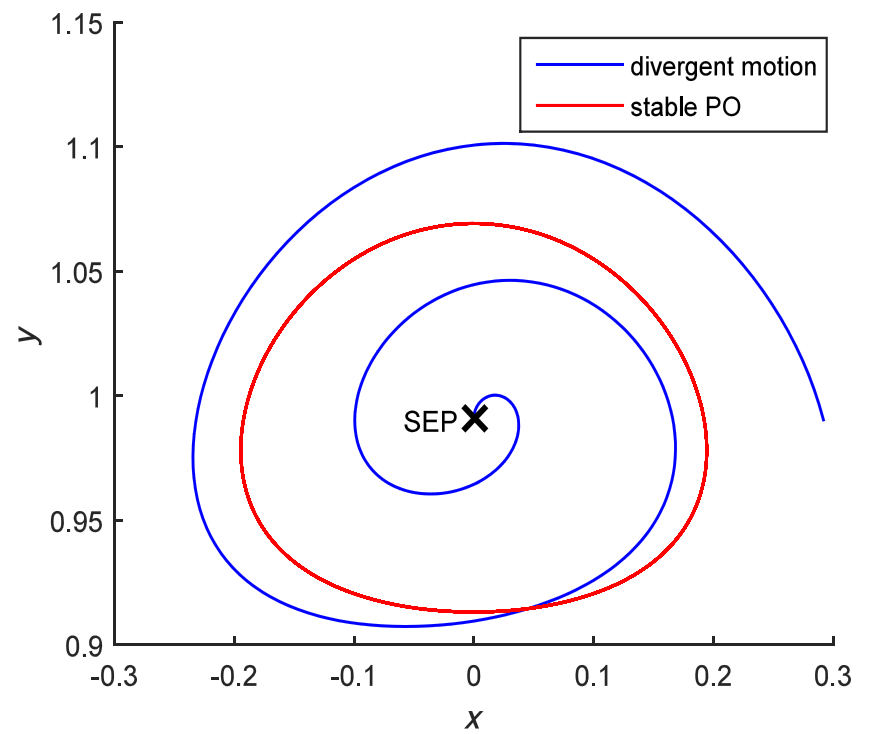

Figure 14. One example of a stable-period PO around the SEP (red curve). The blue line indicates a trajectory starting from the unstable SEP, with an initial perturbation of $10^{-2}$ along the $y$-direction.

while the red trajectory illustrates a stable orbit, which is integrated for the time of amounts of orbital period.

The results in this subsection are just an example to show how the long- and short-period families are organized in the phase space when the SEP is stable (Figure 11) and how the 
a) example vertical orbits

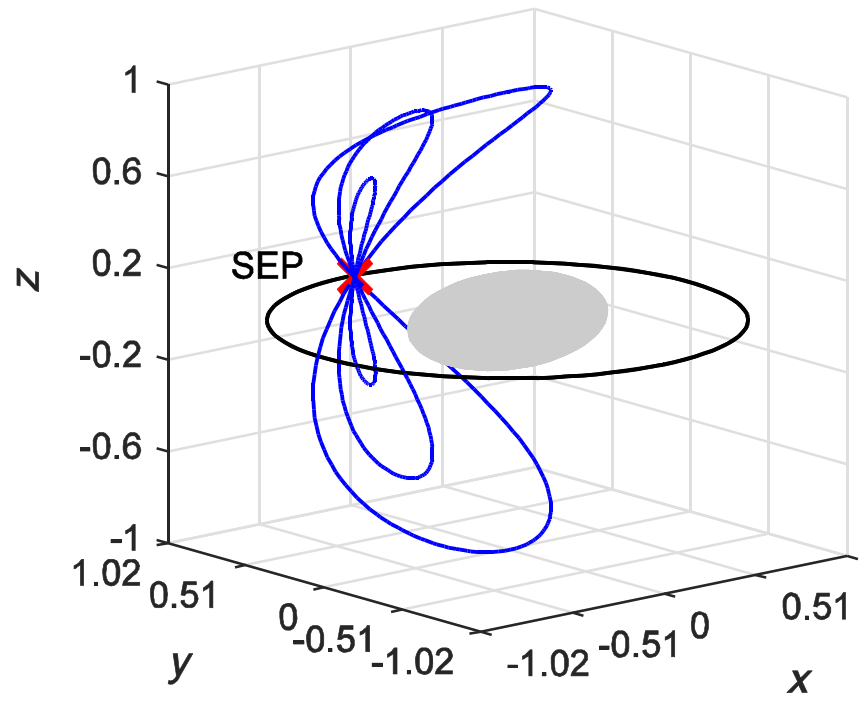

b)

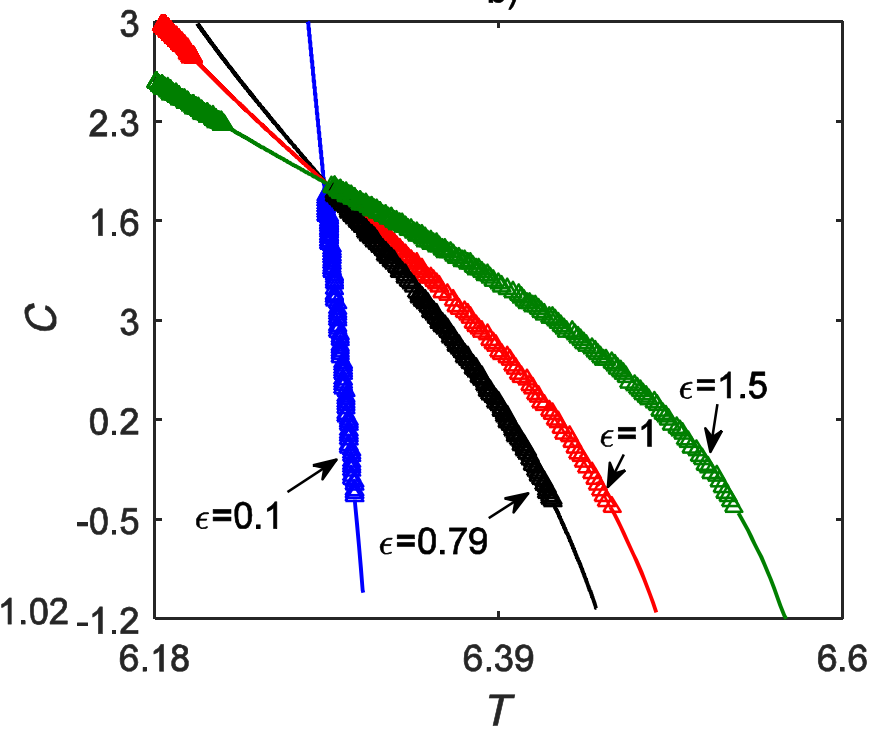

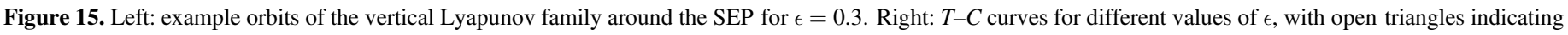
the unstable orbits. The retrograde orbits have smaller Jacobi constants on the $T-C$ plane.

two families combine and detach themselves from the SEP when the SEP is unstable (Figures 12 and 13). Our studies show that similar phenomena appear when other examples are chosen (i.e., asteroids with different shape parameters). This indicates that the global dynamics around the SEP in the 2OD gravitational potential are similar to the global dynamics around the triangular libration points of the CRTBP.

\subsection{The Vertical Lyapunov Family}

For the vertical Lyapunov family around the SEP, Figure 15(a) gives some example orbits with increasing amplitude. Similar to the vertical Lyapunov family around the LEP (see Figure 8), this family starts from infinitesimally small librations around the SEP and ends at the black stable retrograde orbit on the equatorial plane traveling twice. The maximum $z$ component first reaches a maximum value (corresponding to a polar 1:1 spin-orbit resonance orbit in the inertial frame) and then decreases to zero (in the inertial frame, the orbital inclination continues to increase from $90^{\circ}$ to $180^{\circ}$ ). The difference from the orbits in Figure 8 is that the ascending or descending nodes are along the asteroid's short axis in the rotating frame in this case.

Figure 15(b) shows $T-C$ curves for different values of $\epsilon$, starting from infinitesimal librations around the SEPs (with larger Jacobi constants) and terminating on a planar retrograde orbit (with smaller Jacobi constants). The open triangles in Figure 15(b) indicate the unstable orbits of this family. There are several features of these $T-C$ curves. (1) For large $\epsilon$ values, an unstable region appears for orbits close to the SEPs. This is understandable because, according to the study of Section 4.2, the SEPs become unstable for large $\epsilon$ values. (2) Orbits with inclinations close to $90^{\circ}$ are unstable. Our studies indicate that this phenomenon is closely connected with the joint effects of the 1:1 resonance and the well-known frozen orbits. We have found similar phenomena for other spatial resonance families. A detailed explanation will be given in a coming paper. (3) Retrograde orbits have much better stability properties and can exist even for very large 2OD terms.

\section{Conclusion}

This work is devoted to describing the global dynamics of the EPs in the rotating frame of a 2OD gravitational potential by studying the genealogy and stability of the periodic families generated from them. With asteroids as our research targets, the $2 \mathrm{OD}$ terms are much larger than those for major bodies in the solar system. For the LEPs, invariant manifolds are also studied. Since the orbits around the EPs in the rotating frame can also be viewed as orbital motions in the inertial frame that are in 1:1 resonance with the asteroid's rotation, this study can also be taken as a treatment of the $1: 1$ resonance orbits.

For the LEPs, the following is found. (1) The planar Lyapunov family is generally unstable; but, for small 2OD terms, stable planar Lyapunov orbits with large amplitudes exist. They are highly eccentric 1:1 resonance orbits in the equatorial plane, with the periapse and apoapse aligning along the long axis of the asteroid. (2) For large 2OD terms, spatial POs generated from the planar Lyapunov family also exist. (3) The reachable region of invariant manifolds associated with the LEP is highly constrained by the inner and outer spin-orbit resonances, a phenomenon similar to the CRTBP. (4) The vertical Lyapunov family is generally unstable. This family initiates from the LEP and ends at a planar retrograde orbit traveling twice. If the 2OD terms are large, the genealogy of this family changes (see Figure 8).

For the SEPs, the following is found. (1) For a stable SEP, the planar short-period orbit is generally stable and becomes unstable for a large-amplitude orbit. The planar long-period family terminates on a planar short-period orbit traveling $N$ times, where the value of $N$ depends on the two basic frequencies in the linearized model of the planar motions around the SEPs. (2) With the 2OD terms increasing, the SEP evolves from stable to unstable. Meanwhile, the planar 
long- and short-period families merge to form a single family. In this case, even though the SEP itself is unstable, stable planar POs of finite sizes exist around it. (3) The vertical Lyapunov family initiates from the SEP and ends at a planar retrograde orbit traveling twice. The stability of this family is based on the joint effects of the 1:1 resonance and the frozen orbits. A detailed explanation is not given here but will be given in a future paper.

Through these studies, it is found that, in the 2OD gravitational potential, not only are the local dynamics around the EPs equivalent to the libration points of the CRTBP (for example, the LEPs are of the saddle $\times$ center $\times$ center type, which is the same as the collinear libration points, and the SEPs are either stable or complex unstable, which is the same as the triangular libration points), but the global dynamics also show a similarity to those of the CRTBP (for example, the spatial halo family bifurcating from the planar periodic family of the LEPs, the accessible region of the invariant manifolds associated with the LEPs, and the genealogy between the longand short-period families associated with the SEPs). The current study assumes potential applications for asteroids that usually have large 2OD terms. However, since we neglect higher-order nonspherical terms of specific asteroids, the results in this work may have limitations when dealing with orbital motions very close to an asteroid's surface. For example, some asteroids may have more than four EPs outside their bodies (Magri et al. 2007; Wang et al. 2014), and the global dynamics around them are expected to be different. It would be interesting to focus on the global dynamics around the EPs for specific asteroids and compare the results with ours.

This work was supported by the National Natural Science Foundation of China $(11322330,11673072)$ and the National Basic Research Program of China (2013CB834100).

\section{References}

Balmino, G. 1994, CeMDA, 60, 331

Broschart, S. B., Lantoine, G., \& Grebow, D. J. 2014, CeMDA, 120, 195
Ceccaroni, M., \& Biggs, J. 2013, Icar, 224, 74

Chauvineau, B., Farinella, P., \& Mignard, F. 1993, Icar, 105, 370

Conley, C. 1968, SJAM, 16, 732

Delsate, N. 2011, P\&SS, 59, 1372

Elipe, A., \& Lara, M. 2004, JAnSc, 51, 391

Feng, J., Noomen, R., Hou, X., Visser, P., et al. 2017, CeMDA, 127, 67

Feng, J., Noomen, R., Visser, P., et al. 2015, Ap\&SS, 357, 124

Fujiwara, A., Kawaguchi, J., Yeomans, D., et al. 2006, Sci, 312, 1330

Geissler, P., Petit, J. M., Durda, D. D., et al. 1996, Icar, 120, 140

Gómez, G., Koon, W. S., Lo, M. W., et al. 2004, Nonli, 17, 1571

Henrard, J. 2002, CeMDA, 83, 291

Herrera-Sucarrat, E., Palmer, P. L., \& Roberts, R. M. 2014, JGCD, 37, 907

Hou, X. Y., \& Liu, L. 2009, CeMDA, 104, 241

Howell, K. C., Barden, B. T., Wilson, R. S., et al. 1997, in Spaceflight Mechanics, Advances in the Astronautical Science Series, vol. 95 (San Diego, CA: American Astronautical Society), 1665

Howell, K. C., \& Spencer, D. B. 1986, AcAau, 13, 473

Hu, W., \& Scheeres, D. J. 2002, JGCD, 25, 765

Hu, W., \& Scheeres, D. J. 2004, P\&SS, 52, 685

Jiang, Y., Baoyin, H., Li, J., et al. 2014, ASS, 349, 83

Kaula, W. M. 1966 Theory of Satellite Geodesy: Applications of Satellites to Geodesy (Waltham, Mass: Blaisdell)

Koon, W. S., Lo, M. W., Marsden, J. E., et al. 2000, Chaos, 10, 427

Llibre, J., Martínez, R., \& Simó, C. 1985, JDE, 58, 104

Magri, C., Ostro, S. J., Scheeres, D. J., et al. 2007, Icar, 186, 152

Murray, C. D., \& Dermott, S. F. 1999, Solar System Dynamics (Cambridge: Cambridge Univ. Press)

Press, W. H. 2007, (Cambridge: Cambridge Univ. Press)

Russell, C., Raymond, C., Coradini, A., et al. 2012, Sci, 336, 684

Scheeres, D. J. 1994, Icar, 110, 225

Scheeres, D. J. 1999, CeMDA, 73, 339

Scheeres, D. J. 2012, JGCD, 35, 987

Scheeres, D. J., \& Hu, W. 2001, CeMDA, 79, 173

Scheeres, D. J., Williams, B., \& Miller, J. 2000, JGCD, 23, 466

Siegel, C. L., \& Moser, J. K. 1971, Lectures on Celestial Mechanics (Heidelberg: Springer)

Sierks, H., Barbieri, C., Lamy, P. L., et al. 2015, Sci, 347, aaa1044

Sweeter, T. H., Broschart, S. B., Angelopoulos, V., et al. 2011, SSRv, 165,27

Szebehely, V. 1967, Theory of Orbits: The Restricted Problem of Three Bodies (New York: Academic)

Tricarico, P., \& Sykes, M. V. 2010, P\&SS, 58, 1516

Vasilkova, O. 2005, A\&A, 430, 713

Wang, X., Jiang, Y., \& Gong, S. 2014, Ap\&SS, 353, 105

Werner, R. A., \& Scheeres, D. J. 1997, CeMDA, 65, 313

Xin, X., Scheeres, D. J., \& Hou, X. Y. 2016, CeMDA, 126, 405

Yu, Y., \& Baoyin, H. 2012, MNRAS, 427, 872

Zeng, X., Jiang, F., Li, J., \& Baoyin, H. 2015, Ap\&SS, 356, 29 